\title{
Neutron scattering study of the terminating protons in the basic structural units of non-graphitising and graphitising carbons
}

\author{
P. W. Albers, W. Weber, K. Möbus, S. D. Wieland \\ and S. F. Parker
}

\section{Published version information}

Citation: Albers PW et al. "Neutron scattering study of the terminating protons in the basic structural units of non-graphitising and graphitising carbons". Carbon, vol. 109 (2016): 239-245.

doi: http://dx.doi.org/10.1016/i.carbon.2016.08.007

This version is made available in accordance with publisher policies under a Creative Commons CC-BY-NC-ND licence. Please cite only the published version using the reference above. 


\title{
Neutron scattering study of the terminating protons in the basic structural units of non-graphitising and graphitising carbons
}

\author{
P. W. Albers ${ }^{a^{*}}$, W. Weber ${ }^{\text {a }}$, K. Möbus ${ }^{\text {b }}$, S. D. Wieland ${ }^{\text {b }}$ \\ and S. F. Parker ${ }^{\mathrm{c}}$

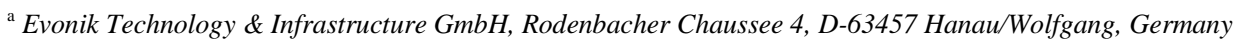 \\ ${ }^{\mathrm{b}}$ Evonik Resource Efficiency GmbH, Rodenbacher Chaussee 4, D-63457 Hanau/Wolfgang, Germany \\ ${ }^{c}$ ISIS Facility, STFC Rutherford Appleton Laboratory, Chilton, Didcot, OX11 0QX, United Kingdom
}

Received ******; accepted ******; *peter.albers@evonik.com

\begin{abstract}
The proton-related fine structure of inelastic neutron spectra of glassy carbon, pure anthracite coal, furnace black and activated carbon has been evaluated using periodic density-functional perturbation theory models of graphene sheets with isolated (H1), vicinal hydrogen (H2) and mixed (H1, H2) termination. Significant differences in the out-of-plane aryl C-H bending/wagging mode region were observed which are not simply numerically correlated with the hydrogen content but with the fine structure of the carbons of different origin and production technology. The basic structural units in glassy carbon type 1 appear to be more uniform than in other carbons, H1 and H2 terminations seems to occur in distinct domains and a mixture of the types is sparse. The neutron spectra of type 2 material and of acetylene blacks of low hydrogen content show similarity to the spectrum of pure graphite, however, transmission electron microscopy reveals distinct differences in morphology at the nanoscale.
\end{abstract}

\section{Introduction}

Glassy carbon (GC) [1,2] mainly consists of $\mathrm{sp}^{2}$ carbon with highly disturbed ordering and stacking [3]. This may imply enhanced surface ruggedness, disorder and differences in size, fine-structure and orientation of the basic structural units (BSU's). Phenolic resin (e.g.) is used as a templating educt material to produce glassy carbon which according to the Jenkins model 'memorises' the molecular orientation of the tangled structure of the precursor polymer [4]. GC's are reported to be nongraphitising and resistant to degradation up to high temperature under inert gas or vacuum conditions. According to the Franklin model [5] it can be assumed that GC consists of smaller BSU's than may be found in pure ordered graphitic matter or other grades of carbon such as coal, carbon blacks of different production technology [6] or activated carbons [7]. These differences could be accompanied by differences in hydrogen content and, correspondingly, varying relative proportions of hydrogenous substitutional patterns down to the nanometre scale [8, and 6].

To improve the understanding of the fundamental differences between graphitising and non-graphitising carbons we have utilised the unique potential of inelastic incoherent neutron scattering (IINS) to selectively study the proton dynamics of carbonaceous matter from different feedstocks and of different hydrogen content. IINS spectra of acetylene-derived blacks with different degrees of ordering and gas and furnace blacks produced from natural gas and oil respectively were compared. Due to the high purity and $\mathrm{sp}^{2}$ character, acetylene black is used as a support material in the production of various hydrogenation catalysts for chemical syntheses. Gas blacks are used as brilliant black pigments and the furnace blacks as reinforcing fillers for tyres. High purity noble anthracite coal (Sophia Jacoba mine), activated carbon derived from beech wood and graphite as a reference material for background subtraction were included in this study. Transmission electron microscopy (TEM) at enhanced resolution revealed pronounced differences in the fine structure of these carbons.

According to the model derived from transmission electron microscopy (TEM) the basic structural units of carbon blacks and other finely divided graphitising carbons consist of small graphite-like platelets which are arranged in turbostratic disorder to form spherical structures [6]. These entities must be terminated by (few) oxygen-, nitrogen- or sulphur-containing functional groups and, predominantly, by protons [8] - otherwise these flat open $\mathrm{sp}^{2}$ structures would not be stable and fullerenes or nanotubes would be formed. Curvature is induced by nonhexagonal rings, e.g. corannulene-type entities [9]. Due to the absorption of electromagnetic radiation and the electrical conductivity of such finely divided carbons, these protons in edge termination can be studied exclusively by inelastic neutron scattering [10]. Carbon blacks can be graphitised to different degrees, whereas glassy ("vitreous") carbons, which are used as electrode materials, in electronics and construction, and as crucibles, are known to be resistant to graphitisation.

Recently we have investigated the proton dynamics of type 1 GC [11] (maximum service temperature under argon: $1000^{\circ} \mathrm{C}$ ) on TOSCA [12, 13]. The IINS 
spectrum showed a well separated sharp band in the out-ofplane aryl $\mathrm{C}-\mathrm{H}$ bending/wagging region which can be assigned to two adjacent hydrogen entities in $\mathrm{sp}^{2}$ carbons, structures as dominating in the coronene molecule [14]. This indicates quite similar and uniform properties of the BSU's in this synthetic grade of carbon derived from resin. Compared to GC IINS data on other carbons [10] revealed that the width of the out-of-plane vibrational mode region often is much broader and its fine structure more complicated and less resolved in various grades of coals $[15,16]$, activated carbons [14], diesel soots [17], carbon blacks [18] or technical cokes deposited or transformed on industrial catalysts [19]. This may indicate that such graphitising carbons are formed by BSU's of a much broader or more irregular size distribution compared to non-graphitising GC's and it may be presumed that this translates to different relative proportions of terminating protons (isolated/ vicinal/ ternary/quaternary). To probe this in more detail, a set of neutron spectra of carbons with comparable resolution were recorded on TOSCA and evaluated by computational methods focusing on isolated and vicinal bound protons in graphene planes which were used in a first approximation to model BSU's and to check the feasibility of this approach in evaluating the inelastic neutron spectra of carbons.

\section{Experimental}

\subsection{Materials}

GC-powder and -plate samples of type 1 and type 2 specification were compared whose maximum service temperature under argon or in vacuum is defined as 1000 or $3000 \mathrm{~K}$, respectively. The samples 1-5 (Table 1) were purchased from Alfa Aesar ${ }^{\circledR}$. High purity anthracite coal (> 95\% C) was obtained from the mine Sophia Jacoba (Hückelhoven-Ratheim, Germany). A steam activated carbon was derived from beech wood; the nitrogen surface area is $>1000 \mathrm{~m}^{2} \mathrm{~g}^{-1}$ and the total pore volume $>1.3 \mathrm{ml} \mathrm{g}^{-1}$. The surface area of the non-porous gas black and the furnace black are 320 and $35 \mathrm{~m}^{2} \mathrm{~g}^{-1}$, the acetylene blacks are 70 and $80 \mathrm{~m}^{2} \mathrm{~g}^{-1}$.

The integral hydrogen content was determined with a LECO TCH600 instrument. The sample weights used in the individual IINS experiment are noted in Table 1 indicating that the observed differences in proton dynamics (section 3.2) are significant and averaged at the macroscopic level over bulk quantities of carbons.

Sample 8 was measured by means of TEM as received and after subsequent graphitisation in an electro-furnace under argon gas.

\subsection{Transmission electron microscopy}

A Jeol 2010F field emission transmission electron microscope (FE-TEM) was operated at $200 \mathrm{keV}$ acceleration voltage. A 4k x 4k F416 TemCam (TVIPS) was utilised.
Table 1

Carbon materials, hydrogen content and sample weight in the IINS sample can.

\begin{tabular}{lll} 
& Hydrogen content & $\begin{array}{l}\text { IINS } \\
\text { sample } \\
\text { weight }\end{array}$ \\
& & $\mathrm{g}$ \\
\cline { 2 - 3 } & $\mu \mathrm{g} \mathrm{g}^{-1}$ & 15.25 \\
\hline 1 Glassy carbon plate type 1, 4 mm & $4100 \pm 200$ & 14.66 \\
2 Glassy carbon plate type 2, 4 mm & $60 \pm 20$ & 47.81 \\
3 Glassy carbon powder type 1, 0.4-12 & $5500 \pm 200$ & \\
micron & & 47.14 \\
4 Glassy carbon powder type 2, 0.4-12 & $950 \pm 200$ & \\
micron & & 107.2 \\
5 Graphite rod & $30 \pm 10$ & 6.36 \\
6 Acetylene black powder & $273 \pm 30$ & 8.67 \\
7 Acetylene black powder & $305 \pm 30$ & 41.28 \\
8 Furnace black N660 ${ }^{1)}$ & $2100 \pm 200$ & 8.87 \\
9 Gas black FW1 powder & $4100 \pm 300$ & 16.64 \\
10 Noble anthracite (bulk piece) & $2990 \pm 200$ & 36.50 \\
11 Activated carbon pellets & $2300 \pm 20$ & \\
& & \\
\hline
\end{tabular}

1) After graphitisation $200 \pm 30 \mu \mathrm{g} \mathrm{g}$

\subsection{Inelastic incoherent neutron scattering}

The powder-shaped carbons and the porous pelletized activated carbon sample 11 were pumped down in their individual IINS sample can for $24 \mathrm{~h}$ at room temperature using a turbomolecular pump and sealed by a welded bellow valve (Nupro).

IINS spectra were recorded using the spectrometer TOSCA [12, 13] at the spallation neutron source ISIS. A sample can was quenched in liquid nitrogen, loaded in a closed cycle helium cryostat and measured at $20 \mathrm{~K}$. A reference measurement to verify the presence of traces of water in a glassy carbon sample (type 1) and the activated carbon were recorded using the MAPS spectrometer [20].

\subsection{Computational methods}

Periodic density functional theory (periodic-DFT) studies of the hydrogen termination of graphene planes was carried out using the plane-wave pseudopotential program method as implemented in CASTEP [21, 22]. The planewave cut-off energy was $900 \mathrm{eV}$ and exchange and correlation were approximated using the GGA PBE functional. Brillouin zone sampling of electronic states was performed on $10 \times 8 \times 1,10 \times 12 \times 1$ and $4 \times 8 \times 1$ MonkhorstPack grids for the [100], [110] and [310] systems respectively. The equilibrium structure, an essential prerequisite for lattice dynamics calculations was obtained by BFGS geometry optimization after which the residual forces were converged to zero within $\pm 0.006 \mathrm{eV} \AA^{-1}$. Phonon frequencies were obtained by diagonalisation of dynamical matrices computed using density-functional perturbation theory [23]. For the [110] system, the dispersion across the entire Brillouin zone was calculated. The resulting dispersion curves were almost flat for the hydrogen modes. We note that the unit cells are sufficiently large that, relative to graphene, the Brillouin zone is wellsampled. The atomic displacements in each mode that are part of the CASTEP output, enable visualization of the modes to aid assignments and are also all that is required to generate the IINS spectrum using the program ACLIMAX [24]. ACLIMAX uses the CASTEP output to generate an 
INS spectrum that has the same $(Q, \omega)$ trajectory as TOSCA. The program calculates all the transitions up to 0 $\rightarrow 10$. Any value may be used for the Debye-Waller factor, in this case, we used the default value of the exponent of $0.035 \AA^{2}$. In the present instance of a periodic calculation on an extended solid, the distinction between internal and external modes is lost and all the modes are "internal". Thus the standard calculation of overtones and combinations automatically includes the phonon wings. It is emphasised that for all the calculated spectra shown the transition energies have not been scaled.

The structures considered were generated from the graphite crystal structure by cleavage along [100],[110], and [310] directions and hydrogen terminated top and bottom. To reduce the computational expense, the periodicity along the interlayer $c$ direction was removed. The resulting twodimensional slabs were separated by a $10 \AA$ vacuum gap. This had the side effect of reducing the restoring force perpendicular to the planes, thus some of the low energy, $<100 \mathrm{~cm}^{-1}$, modes had much greater intensity than they would have had in graphite. These modes were set to have near-zero intensity. The thickness of the slab was investigated, it was found that 10 layers were needed for the [100] system to ensure that there was no interaction between the hydrogens at the top and the bottom of the layer. For the [110] and [310] systems seven layers were sufficient.

\section{Results and Discussion}

\subsection{Transmission electron microscopy}

The TEM images in Fig. 1 a-d illustrate differences between the glassy carbon type 1 and type 2 . The type 1 material (1000 K operation) is highly disordered and of amorphous appearance ("vitreous carbon" [1,2 ]) whereas the type 2 (3000 K operation) material shows some graphitic ordering in the topmost surface region whereas in near-surface regions similarity to type 1 material is observed. The graphitic ordering in the surface region of the type 2 material (Fig. 1 d) seems slightly enhanced compared to a GL-200 glassy carbon sample [3].
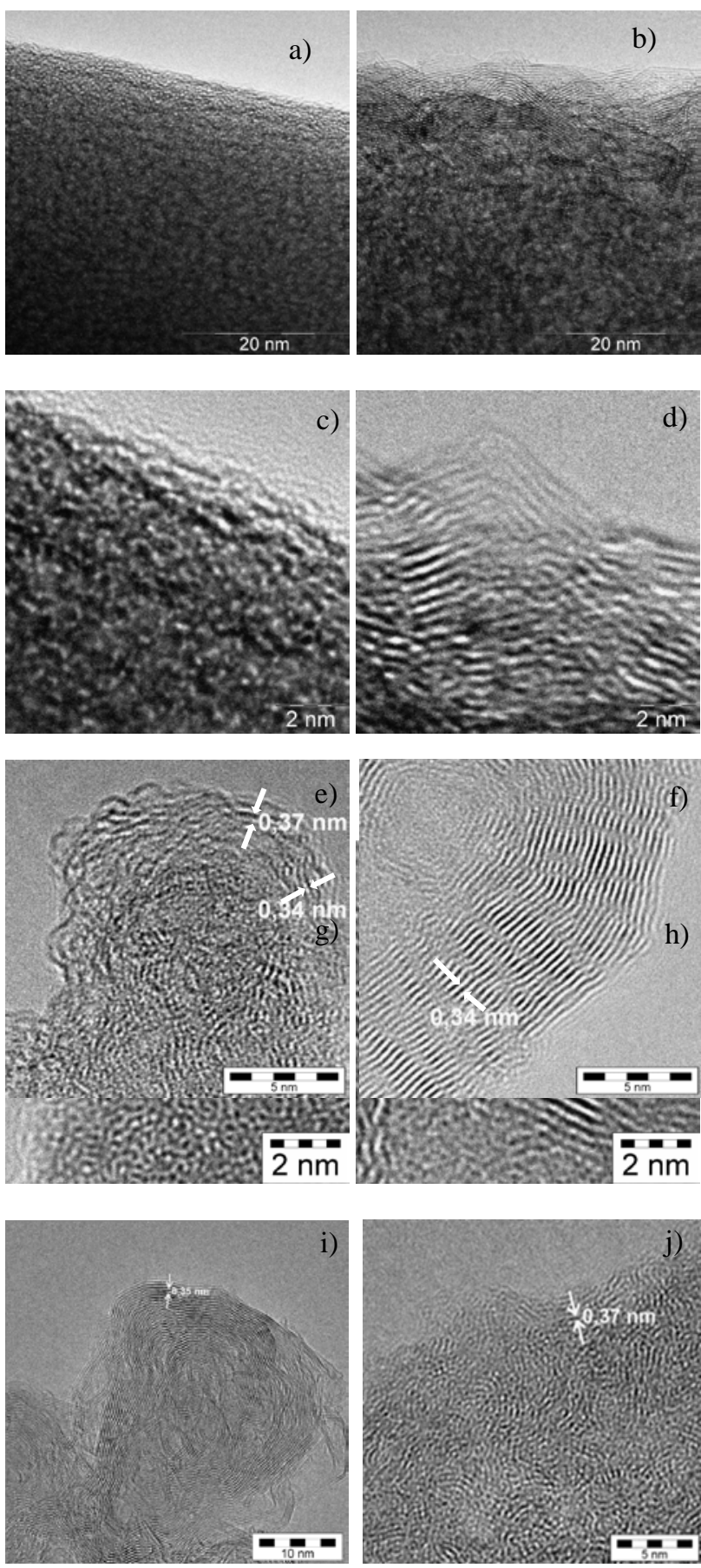

Fig. 1. Top to bottom: a-d) Comparison of glassy carbon's surface and selvedge region; left: type 1, right: type 2; e-f) furnace black before (left) and after (right) high temperature treatment under argon; g) noble anthracite coal (Sophia Jacoba mine); h) graphite rod; i-j) acetylene blacks sample 6 (left) and sample 7 (right) (Table 1).

Different fine-structure is observed for carbon black: the paracrystalline microstructure appears (Fig. 1 e) according to the model described in [6 p. 102] and the thermal posttreatment leads to enhanced graphitic ordering and the 
decrease of the d-values between the graphitic sheets from $0.37 \mathrm{~nm}$ to $0.34 \mathrm{~nm}$ [25]. The noble anthracite coal shows even more disorder $(0.36 \mathrm{~nm}-0.34 \mathrm{~nm})$ whereas the graphite reference (2990 vs. $30 \mathrm{hg} \mathrm{g}^{-1} \mathrm{H}$, Table 1 ) shows enhanced two-dimensional $\mathrm{sp}^{2}$-ordering (Fig. $1 \mathrm{~g}-\mathrm{h}$ ) compared to the topmost surface region of the type 2 GCplate (Fig. $1 \mathrm{~d}$ ). The acetylene blacks of slightly higher but similar hydrogen content are partly ordered and partly paracrystalline/disordered and the d-values show small variations accordingly (0.35 to $0.37 \mathrm{~nm})$.

\subsection{Inelastic incoherent neutron scattering}

Fig. 2 and 3 show pronounced differences in the properties of GC carbon type 1 powder and plate whereas the spectral features of type 2 material are quite similar for both, powder and plate. The out-of-plane C-H deformational modes of the type 1 plate are much sharper than for the powder material which suggests some similarity to the spectrum of activated carbon (Fig. 4). The difference spectrum between powder and plate in Fig. 3 shows the presence of traces of water in the material indicated by the characteristic shape, position and broadness of the librational modes of ice [26, 27] (please note that the IINS measurements are recorded at low temperature).

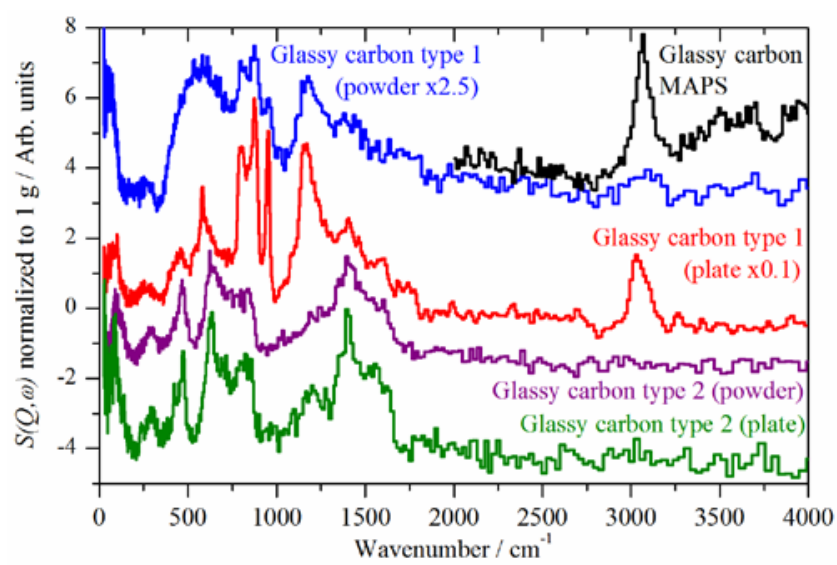

Fig. 2. IINS spectra of type 1 and type 2 quality GC plates and powders

This conclusion is supported by an additional measurement (after drying the sample at $105^{\circ} \mathrm{C}$ for $12 \mathrm{~h}$ ) using the MAPS spectrometer (Fig. 2) which allows complementary access and signal assignment to the $\mathrm{O}-\mathrm{H}$ modes. A weak, broad $\mathrm{O}-\mathrm{H}$ stretch mode is apparent at $\sim 3600 \mathrm{~cm}^{-1}$ confirming the assignment of the $\sim 500 \mathrm{~cm}^{-1}$ band to water.

Comparison of the spectra of both the high temperature type 2 GC's (Fig. 2) and the graphite rod (Fig. 3) suggests some similarity. With respect to the low hydrogen levels (Table 1, 60/950/30 $\mathrm{g} \mathrm{g} \mathrm{g}^{-1} \mathrm{H}$ ) hydrogenous motions are largely missing. In spite of the different fine structures seen in the TEM (Fig. $1 \mathrm{a}-\mathrm{d}, \mathrm{h}$ ) and that nongraphitising material (or material that only shows some traces of surface-graphiticity, type 2 plate) is compared with graphitic carbon this similarity is striking: GC's of type 2 at the given low hydrogen content can also not accommodate lattice dynamics at higher frequencies than pure graphite and the out-of-plane and in-plane aryl $\mathrm{C}-\mathrm{H}$ wagging modes which dominate the IINS spectra of the GC's type 1 are largely missing. The structural evolution of GC's [28, 29] with temperature due to the loss of hydrogenous species is clearly and sensitively indicated by the changes in the low energy vibrational mode region below ca. $1800 \mathrm{~cm}^{-1}$ (Fig. 2).

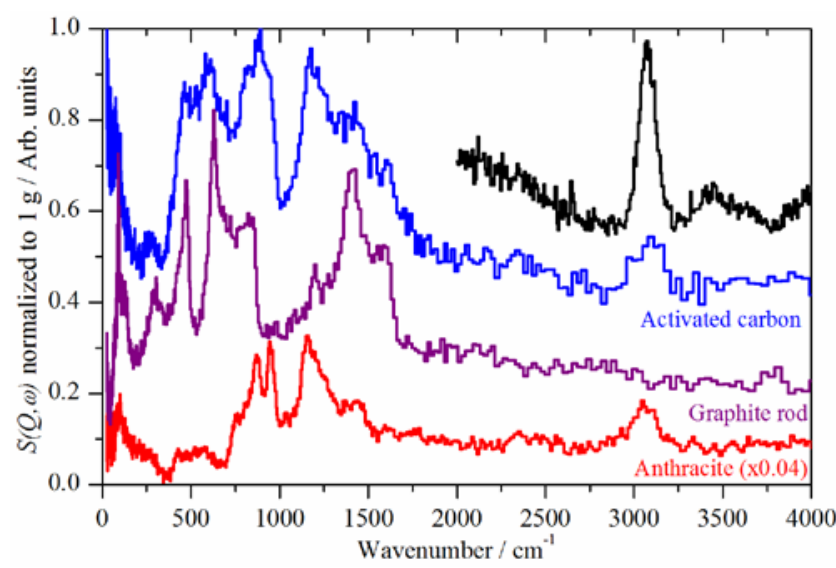

Fig. 3. IINS spectra of carbons. Top to bottom: activated carbon, graphite rod and Sophia-Jacoba anthracite. The inset shows the high energy region of the activated carbon sample recorded on MAPS.

Also the features around $500 \mathrm{~cm}^{-1}$ in the spectrum of the activated carbon (Fig. 3) could be assigned to either graphitic material or absorbed water. From subtractions of the graphite rod and the carbon black N660 (Fig. 4) from the spectrum, it is clear that the carbon black is the better model and that absorbed water is present in the porous material of high surface area. This supported by the MAPS data, which shows an $\mathrm{O}-\mathrm{H}$ stretch at $3460 \mathrm{~cm}^{-1}$.

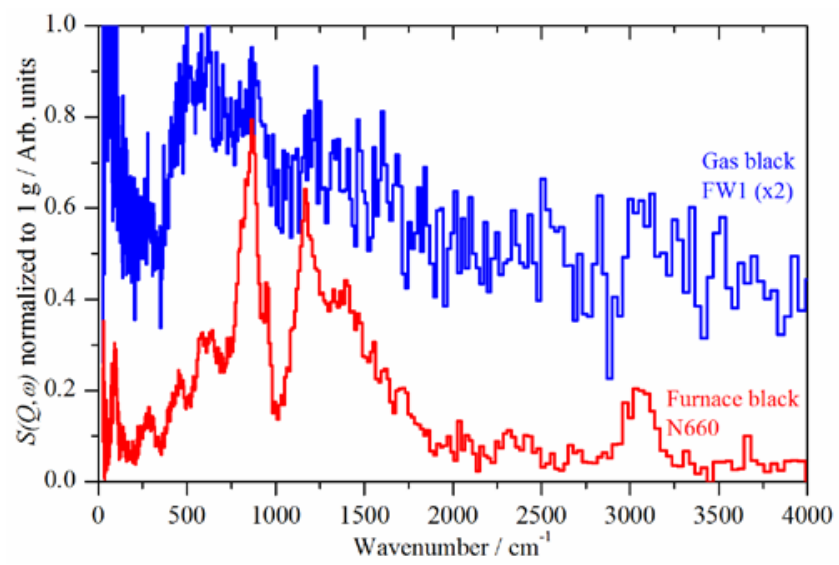

Fig. 4. IINS spectra of carbon blacks: gas black FW1 (upper trace) and carbon black N660 (lower trace).. 
The IINS spectra of the graphite rod (Fig. 3 ) and the acetylene blacks of different hydrogen content (Fig. 5) (Table 1, 30 vs. 273 and $305 \mu \mathrm{g} \mathrm{g}^{-1} \mathrm{H}$ ) are still more similar to each other ( Fig. 4) than to furnace black (Fig. 4) and gas black (Fig. 4) and to activated carbon (Fig. 3). The spectrum of the high surface area gas black FW1 in Figure 4 is dominated by the presence of adsorbed water and this is confirmed by the subtraction of the spectrum of the lower surface area furnace black N660.

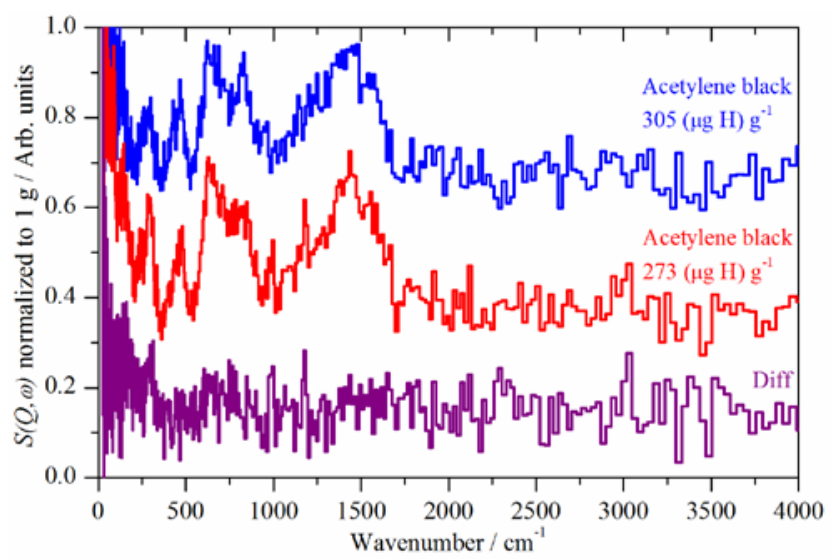

Fig. 5. IINS spectra of acetylene blacks and difference spectrum.

It is of interest to see if the adsorbed/absorbed water varies between the different types of carbons: GC, porous carbon and high surface area gas black. Fig. 6 compares the spectra of the water obtained from the subtractions. Apart from the artefact in the spectrum from the activated carbon due to a miscancellation, the spectra are very similar. In particular, the leading edge of the librational bands is at $\sim 430 \mathrm{~cm}^{-1}$ in all three samples, indicating that the intermolecular $\mathrm{O} \cdots \mathrm{O}$ distance is the same in all three materials and larger than in ice $\mathrm{I}_{\mathrm{h}}$. The sloping leading edge indicates that the water is disordered $[26,27]$. The adsorbed water is not removed by pumping under high vacumm and in the case of the GC type 1 powder not even by high vacuum at $105{ }^{\circ} \mathrm{C}$ overnight. That the structure differs from bulk ice $\mathrm{I}_{\mathrm{h}}$ is indicative of a significant interaction between the water and the surface of the carbon. The disorder is also different from that found in high density amorphous (HDA) ice [26], Fig. 6. It can be seen that both the librational and acoustic translational modes are at lower energy than HDA ice $\left(465 \mathrm{~cm}^{-1}\right.$ for the mid-point of the librational edge and $78 \mathrm{~cm}^{-1}$ respectively). Together with the relatively high energy of the $\mathrm{O}-\mathrm{H}$ stretch modes, $\sim 3500 \mathrm{~cm}^{-1}$, seen in the glassy carbon and the activated carbon samples, the spectra suggest an open network of water molecules with relatively weak hydrogen bonding present.

Previous work had shown that controlled gasphase oxidation of a corresponding high surface area gas black caused an additional vibrational feature at about 410 $\mathrm{cm}^{-1}$ due to enhanced porosity by erosion with ozone [30]. This feature is not observed in the non-oxidised material.

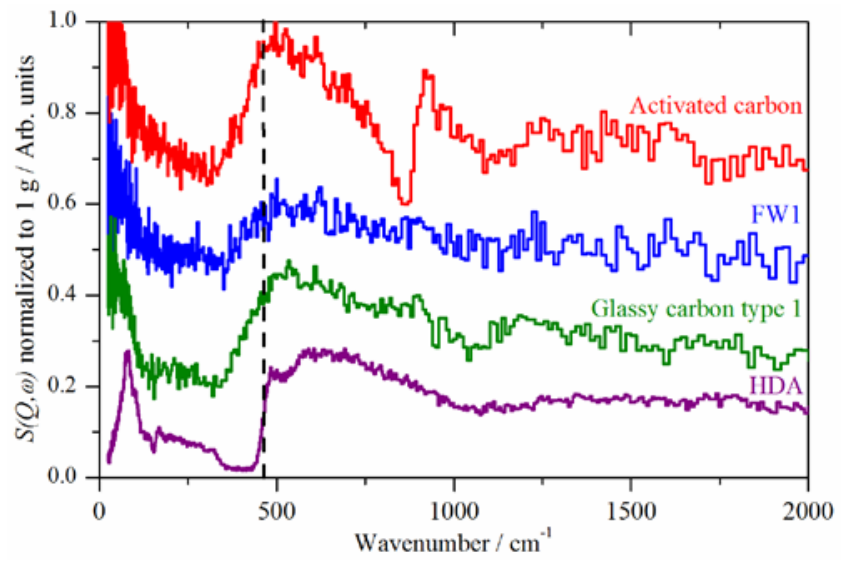

Figure 6: Comparison of adsorbed/absorbed water on carbons. Top to bottom: activated carbon, gas black FW1, dried type 1 glassy carbon powder and high density amorphous (HDA) ice. The dashed vertical line marks the mid-point of the librational edge of HDA ice.

\subsection{Computational results}

The nature of the edge termination of the graphene planes is of considerable interest. We have previously investigated this by generating molecules with one (H1), two (H2) or three (H3) adjacent hydrogens and calculating their vibrational spectra [14]. This approach has been adapted and extended to larger model systems [31]. An alternative method is to create a surface with appropriate termination and to use periodic-DFT to model it. Figure 7 shows a graphene plane cleaved along [100] (1a), [110] (1b) and [310] (1c) to generate $\mathrm{H} 1, \mathrm{H} 2$ and mixed $\mathrm{H} 1, \mathrm{H} 2$ terminated surfaces respectively.

The IINS spectrum of the type 1 glassy carbon plate is compared to those generated from the [100] and [110] systems in Figure 8 a-c. It can be seen that together, the models for $\mathrm{H} 1$ and $\mathrm{H} 2$ termination account reasonably well for all the spectral features. By comparison to the $\mathrm{C}-\mathrm{H}$ stretching mode at $3030 \mathrm{~cm}^{-1}$, we estimate that $\mathrm{H} 1$ and $\mathrm{H} 2$ terminations are present in approximately equal numbers. To model this, the [310] system was created which has the same number of $\mathrm{H} 1$ and $\mathrm{H} 2$ hydrogen atoms. It can be seen, Figure 12 (d), that this is a very poor model of the system, neither the profile nor the relative intensities of the out-ofplane deformation modes around $700-1000 \mathrm{~cm}^{-1}$ is wellreproduced. This would indicate that $\mathrm{H} 1$ and $\mathrm{H} 2$ terminations occur as distinct domains and that a mixture of the types is sparse, at least in glassy carbon. 

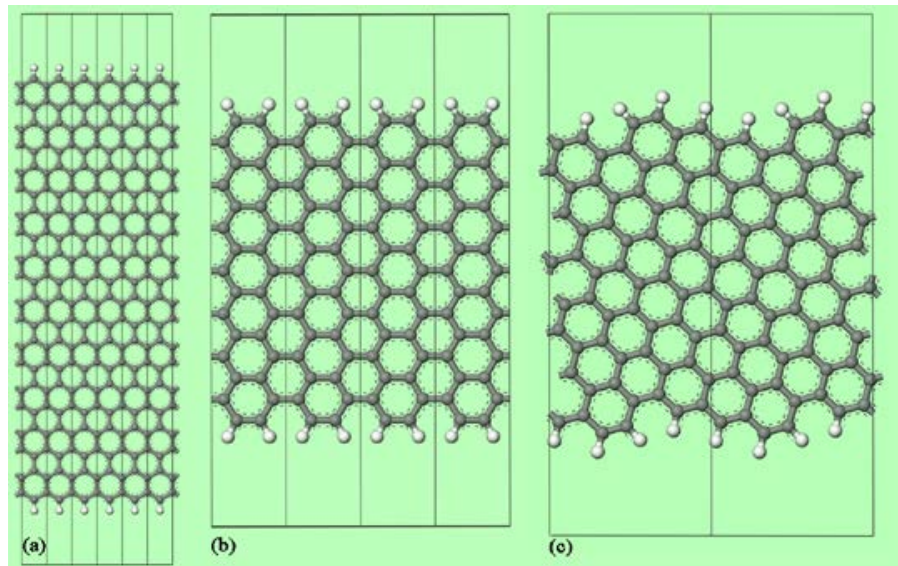

Fig. 7. A graphene plane cleaved along: (a) [100] showing six unit cells, (b) [110] showing four unit cells and (c) [310] showing two unit cells to generate H1, H2 and mixed H1 H2 terminated surfaces respectively.

Based on this analysis, for the materials investigated here, that contain a significant amount of hydrogen (Table 1), we suggest that: type I glassy carbon has $\mathrm{H} 1$ and $\mathrm{H} 2$ termination in a 2:1 ratio, (equal numbers of $\mathrm{H} 1$ and $\mathrm{H} 2$ hydrogens), furnace black N660 has H1 and H2 termination in a 4:1 ratio (twice as many $\mathrm{H} 1$ as $\mathrm{H} 2$ hydrogens), activated carbon has $\mathrm{H} 1$ and $\mathrm{H} 2$ termination in a 2:1 ratio (equal numbers of $\mathrm{H} 1$ and $\mathrm{H} 2$ hydrogens) and noble anthracite coal has $\mathrm{H} 1$ and $\mathrm{H} 2$ termination in a 1.5:1 ratio (one-third more of $\mathrm{H} 2$ than $\mathrm{H} 1$ hydrogens), Table 2.

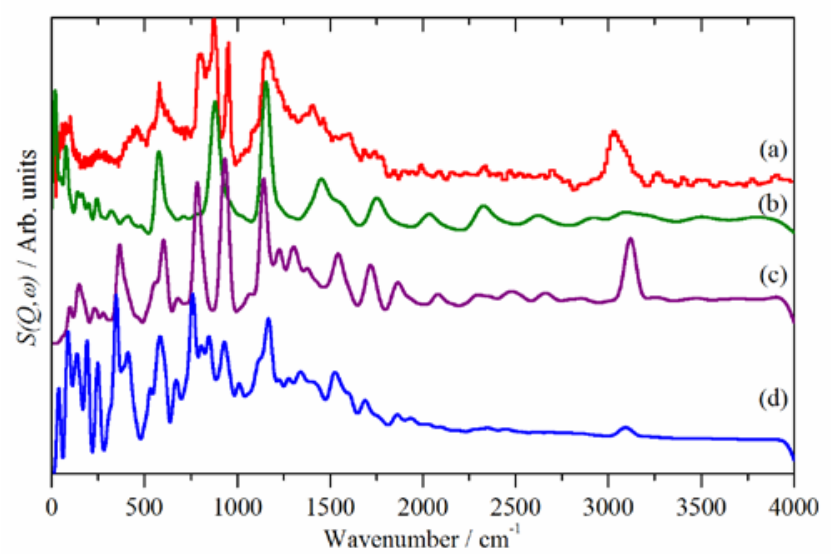

Fig. 8. INS spectra of: (a) type 1 glassy carbon plate, and those generated from a graphene plane cleaved along: (b) [100] to give $\mathrm{H} 1$ termination, (c) [110] to give H2 termination and (d) along [310] to give a mixed H1, H2 (1:1) system.

Figure 9 compares the spectra generated from the models shown in Figure 7 with the IINS spectrum of type 1 glassy carbon plate. It can be seen that the agreement is encouraging. The spectra shown are calculated only at the $\Gamma$-point in the Brillouin zone. The modes relating to carbon motions $\left(<800 \mathrm{~cm}^{-1}\right)$ are likely to be highly dispersed and inclusion of these would require calculations across the complete Brillouin zone. While this would improve the agreement they are significant calculations and the time required could not be justified.

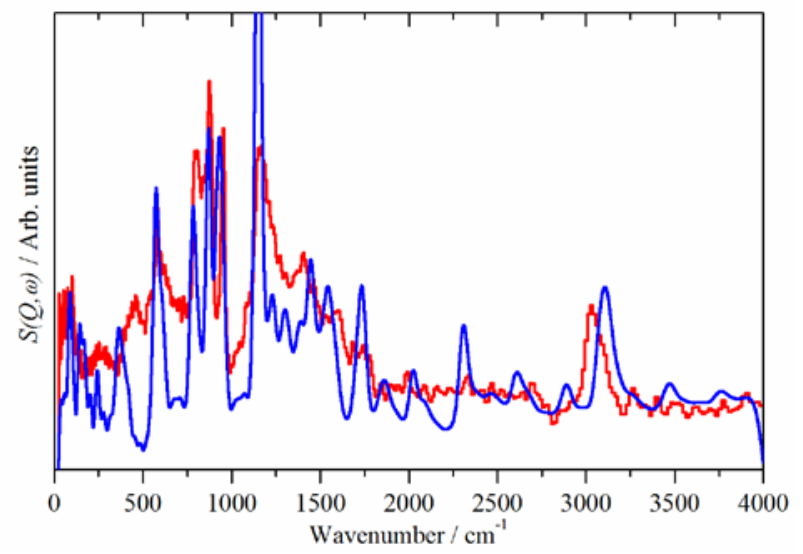

Fig.9. Comparison of the IINS spectrum of type 1 glassy carbon plate (red) and that calculated from a 2:1 $\mathrm{H} 1$ to $\mathrm{H} 2$ mixture (blue).

Table 2.

Calculated and observed transition energies of the out-ofplane $\mathrm{C}-\mathrm{H}$ bending/wagging modes and the $\mathrm{H} 1: \mathrm{H} 2$ ratio.

\begin{tabular}{|c|c|c|c|c|}
\hline & $\mathrm{H} 1 / \mathrm{cm}^{-1}$ & $\mathrm{H} 21$ & $m^{-1}$ & H1:H2 \\
\hline CASTEP & 880 & 785 & 935 & \\
\hline GC type I plate & 873 & 806 & 954 & $2: 1$ \\
\hline $\begin{array}{l}\text { Noble anthracite } \\
\text { coal }\end{array}$ & 875 & 770 & 950 & $1.5: 1$ \\
\hline Activated carbon & 877 & 821 & 933 & $2: 1$ \\
\hline $\begin{array}{l}\text { Furnace black } \\
\text { N660 }\end{array}$ & 864 & 800 & 950 & $4: 1$ \\
\hline
\end{tabular}

3.4 Discussion and outlook

These results on the differences in hydrogen content (Table 1), micro- and nanostructure (TEM, Figure 1) and, correspondingly, proton dynamics (IINS, Figure 2-6, 8, 9, and Table 2) indicate distinct differences between these carbons of different structural complexity and $\mathrm{sp}^{2} / \mathrm{sp}^{3}$ character.

The agreement between experimental neutron data and models of graphene planes of different hydrogen termination is encouraging and illustrates future potential in studying the proton-related properties as structural probes of the basic structural units in carbons. This will enable an improved understanding of different technical properties due to different feedstocks, origin and synthesis conditions. The observation that the experimental neutron spectrum of a glassy carbon type 1 plate is well-approximated by $\mathrm{H} 1$ and $\mathrm{H} 2$ terminated sheets of graphene but not by a mixture of $\mathrm{H} 1$ and $\mathrm{H} 2$ termination in the same BSU suggests that 
the cross-linked stacked graphene layers in nongraphitising carbon according to the Franklin model [5] consists of distinct domains of equal hydrogen termination: $\mathrm{H} 1$ type hydrogen termination on the one hand and $\mathrm{H} 2$ termination on the other.

Size, shape, orientation and stacking as well as disorder, turbostratic arrangement and curvature of BSU's and the type of terminating protons and $\mathrm{H} 1 / \mathrm{H} 2$ ratios as usable properties of carbons are of relevance in many fields of application such as:

1) the reinforcement of rubber materials - the interactions between the hydrogen terminated edges of the surfaces of carbon blacks and the polymer chains

2) lithium ion cells - the correlation between hydrogen content and capacity for reversible lithium insertion in carbons and preferential bonding in the vicinity of the hydrogen atoms [32,33]

3) catalysis - the preferential adsorption, deposition and particle growth of precious metal nanoparticles in edge decoration in technical catalysts [34] indicating interactions between terminating hydrogen in carbons with precious metal atoms on carbon blacks similar to the lithium ions in glassy carbon [33].

\section{Conclusions}

The inelastic neutron spectra of glassy carbons of type 1 and type 2 show distinct differences which directly illustrates structural evolution [28, 29] with temperature and loss of hydrogen. The neutron spectra of type 2 GC and acetylene blacks show similarity to the spectrum of pure graphite, however, TEM reveals pronounced differences of the micro-/nanostructures.

In GC type 1 powder, highly porous activated carbon and a high surface area gas black sample residual adsorbed/absorbed water was observed which is of disordered structure in the ice-like-state.

The out-of-plane aryl $\mathrm{C}-\mathrm{H}$ bending/wagging modes of type 1 GC appear as sharp and well-resolved signals compared to the broader and less-structured bands in the spectra of various coals, carbon blacks, activated carbons or technical cokes observed here and seen previously [10]. This may be attributed to a more regular size and shape distribution of the basic structural units in non-graphitising carbons. This conclusion is supported by DFT-calculations of graphene sheets in evaluating the relative proportions of isolated $\mathrm{H} 1$ and vicinal $\mathrm{H} 2$ termination in analysing the IINS spectra of type 1 GC, furnace black, activated carbon and noble anthracite coal. No simple correlation with total hydrogen content of the carbons is found but with the fine structure according to grade and production technology.

\section{Acknowledgements}

The STFC Rutherford Appleton Laboratory is thanked for access to neutron beam facilities. Computing resources (time on the SCARF computer cluster for the CASTEP calculations) was provided by STFC's e-Science facility. Prof. H. Offermanns (Hanau) is gratefully acknowledged for many inspiring discussions on noble anthracites and other carbons and for supplying the Sophia Jacoba sample.

\section{References}

[1] J.C. Lewis, B. Redfern, F.C. Cowlard, Vitreous carbon as a crucible material for semiconductors, Solid-State Electronics 6 (3) (1963) 251-254.

[2] F.C. Cowlard, J.C. Lewis, Vitreous carbon - A new form of carbon, Journal of Materials Science 2 (6) (1967) 507-512

[3] P.J.F. Harris, Fullerene-related structure of commercial glassy carbons, Phil. Magazine 84 (29) (2004) 3159-3167.

[4] G.M. Jenkins, K. Kawamura, L.L. Ban, Formation and structure of polymeric carbons, Proceedings of the Royal Society London, A327 (1972) 501-517.

[5] R.E. Franklin, Crystallite growth in graphitizing and non-graphitizing carbons, Proceedings of the Royal Society of London, A 209 (1951) 196-218.

[6] J.-B. Donnet, R.C. Bansal, M.-J. Wang, (eds.) Carbon Black Science and Technology, Marcel Dekker, New York, 1993, pp. 95 and 101.

[7] R.C. Bansal, J.-B. Donnet, F. Stoeckli, (eds.) Active Carbon, Marcel Dekker, New York, 1988.

[8] E. Koberstein, E. Lakatos, M. Voll, Zur Charakterisierung der Oberflächen von Rußen und hochdispersen Kieselsäuren, Ber. Bunsenges. Phys. Chem. 75 (10) (1971) 1104-1114.

[9] Y. Hu, O.A. Shenderova, Z. Hu, C.W. Padgett, D.W. Brenner, Carbon nanostructures for advanced composites, Rep. Prog. Phys. 69 (6) (2006) 1847-1895.

[10] P.C.H. Mitchell, S.F. Parker, A.J. Ramirez-Cuesta, J. Tomkinson, Vibrational Spectroscopy with Neutrons: with applications in Chemistry, Materials Science and Catalysis, World Scientific, Singapore, (2005) pp. 487.

[11] S.F. Parker, S. Imberti, S.K. Callear, P.W. Albers, The structure of glassy carbon, Chem. Phys. 427 (2013) 44-48.

[12] D. Colognesi, M. Celli, F. Cilloco, R.J. Newport, S.F. Parker, V. Rossi-Albertini, F. Sacchetti, J. Tomkinson, M. Zoppi, TOSCA neutron spectrometer - the final configuration, Applied Physics A: Materials Science \& Processing 74, Supplement 1 (2002) S64-S66.

[13] S.F. Parker, F. Fernandez-Alonso, A.J. RamirezCuesta, J. Tomkinson, S. Rudic, R.S. Pinna, G. Gorini, J. Fernández Castañon, Recent and future developments on TOSCA at ISIS, J. Phys. Conf. Series 554 (2014) 012003. 
[14] P. W. Albers, J. Pietsch, J. Krauter and S. F. Parker, Investigations of activated carbon catalyst supports from different natural sources, Phys. Chem. Chem. Phys., 5 (2003) 1941-1949.

[15] J. Howard, C.J. Ludman, J. Tomkinson, Inelastic neutron scattering spectra of some English coals: a preliminary study, Fuel 62 (9) (1983) 1097-1100.

[16] F. Fillaux, R. Papoular, A. Lautie, J. Tomkinson, Inelastic neutron-scattering study of the proton dynamics in carbons and coals, Carbon 32 (7) (1994) 1325-1331.

[17] P.W. Albers, H. Klein, E.S. Lox, K. Seibold, G. Prescher,S.F. Parker, INS-, SIMS- and XPS-investigations of diesel engine exhaust particles, Phys. Chem. Chem. Phys 2 (2000) 1051-1058.

[18] P. Albers, K. Seibold, G. Prescher, B. Freund, S.F. Parker, J. Tomkinson, D.K. Ross, F. Fillaux, Neutron spectroscopic investigations on different grades of modified furnace blacks and gas blacks, Carbon 37 (3) (1999) 437444.

[19] P. Albers, S. Bösing, D.K. Ross and S.F. Parker, Inelastic neutron scattering investigations of the products of thermally and catalytically driven catalyst coking, Appl. Catal. A: General 187 (1999) 233-243.

[20] S.F.Parker, D. Lennon, P.W. Albers, Vibrational spectroscopy with neutrons - new directions, Applied Spectroscopy 65 (12) (2011) 1325-1341.

[21] S. J. Clark, M. D. Segall, C. J. Pickard, P. J. Hasnip, M. J. Probert, K. Refson, and M. C. Payne, First principles methods using CASTEP, Z. Krist. 220 (2005) 567-570.

[22] K. Refson, P. R. Tulip, and S. J. Clark, Variational density-functional perturbation theory for dielectrics and lattice dynamics, Phys. Rev. B 73 (15) (2006) 155114.

[23] V. Milman, A. Perlov, K. Refson, S. J. Clark, J. Gavartin, and B. Winkler, Structural, electronic and vibration properties of tetragonal zirconia under pressure: a density functional theory study, J. Phys.: Condens. Matter 21 (2009) 485404 (12 pp).

[24] A. J. Ramirez-Cuesta, Calculation of INS spectra using CASTEP, Comput. Phys. Commun. 157 (3) (2004) 226-238.

[25] GR J. Howe, C. Rawn, L. Jones, H. Ow, Improved crystallographic data for graphite, Powder Diffr. 18 (2) (2003) 159-163. $0.3355300 \mathrm{~nm}$ (100).

[26] J.-C. Li, Inelastic neutron scattering studies of hydrogen bonding in ices, J. Chem. Phys. 105 (1996) 67336755.
[27] J.-C. Li, A.I. Kolesnikov, Neutron spectroscopic investigation of the dynamics of ice, J. Mol. Liq. 100 (2002) 1-39.

[28] F. Rousseaux, D. Tchoubar, Structural evolution of a glassy carbon as a result of thermal treatment between 1000 and $2700^{\circ} \mathrm{C}$ : Tridimensional configuration of a glassy carbon, Carbon 15 (2) (1977) 63-68

[29] J. Yamanaka, E. Yasuda, H. Migita, Y. Tanabe, Cage structure development in furan-resin-derived carbon, Mat. Trans. 42 (3) (2001) 453-456.

[30] P. Albers, A. Karl, J. Mathias, D. K. Ross, S. F. Parker, INS-, XPS- and SIMS-investigations on the controlled post-oxidation of pigment blacks-Detection of different species of strongly adsorbed water, Carbon 39 (2001) 1663-1676.

[31] C. Cavallari, D. Pontiroli, M. Jiménez-Ruiz, A. Ivanov, M. Mazzani, M. Gaboardi, M. Aramini, M. Brunelli, M. Riccò and S. Rols, Hydrogen on graphene investigated by inelastic neutron scattering, J. Phys. Conf. Series 554 (1) (2014) 012009.

[32] Tao Zheng, Yinghu Liu, E. W. Fuller, Sheilla Tseng, U. von Sacken, J. R. Dahn, Lithium insertion in high capacity carbonaceous materials, J. Electrochem. Soc. 142 (8) (1995) 2581-2590.

[33] J. R. Dahn, Tao Zheng, Yinghu Liu, J. S. Xuem Mechanisms for lithium insertion in carbonaceous materials, Science 270 (1995) 590-593.

[34] K. Möbus, E. Grünewald, S. D. Wieland, S. F. Parker, P. W. Albers, Palladium-catalyzed selective hydrogenation of nitroarenes: influence of platinum and iron on activity, particle morphology and formation of $\beta$-palladium hydride, J. Catal. 311 (2014) 153-160. 